\title{
3D modeling of the ASDEX Upgrade edge plasma exposed to a localized tungsten source by means of EMC3-Eirene
}

\author{
T.Lunt ${ }^{\mathrm{a}}$, Y.Feng ${ }^{\mathrm{a}}$, K.Krieger ${ }^{\mathrm{a}}$, A.Kallenbach ${ }^{\mathrm{a}}$, R.Neu ${ }^{\mathrm{a}}$, A.Janzer ${ }^{\mathrm{a}}$, R.Dux ${ }^{\mathrm{a}}$, D.P.Coster ${ }^{\mathrm{a}}$, M.Wischmeier ${ }^{\mathrm{a}}$, N.Hicks ${ }^{\mathrm{a}}$, W.Suttrop ${ }^{\mathrm{a}}$, \\ T.Pütterich ${ }^{\mathrm{a}}$, H.W.Müller ${ }^{\mathrm{a}}$, V.Rohde ${ }^{\mathrm{a}}$, E.Wolfrum ${ }^{\mathrm{a}}$, R.Fischer ${ }^{\mathrm{a}}$, and the ASDEX Upgrade team ${ }^{\mathrm{a}}$ \\ ${ }^{a}$ Max-Planck-Institut für Plasmaphysik, EURATOM Association, Germany
}

\begin{abstract}
We report on simulations carried out by the Edge Monte Carlo 3D (EMC3) - Eirene code package of ASDEX Upgrade divertor discharges exposed to a localized source of $\mathrm{W}$ impurities and compare our results to dedicated experiments. By injecting the impurities first into the divertor and then into the main chamber while observing the impact on the impurity concentration in the core we can determine the 'divertor retention' R, i.e. the capability of the divertor to screen the core from impurities. The simulations show a strong dependence on the strike point - source distance and on collisionality. Experiments with a massive introduction of W with a melting $\mathrm{W}$ pin in the divertor and a moderate injection of $\mathrm{W}$ in the main chamber yield a value of $R \sim 25$ which seems to be consistent with the simulation.
\end{abstract}

Keywords: T1000, T0100, D0500, F0400, I0100

\section{Introduction}

Due to the fact that the power radiated by impurities in a plasma depends strongly on the atomic number, high $\mathrm{Z}$ impurities can significantly reduce the energy confinement of fusion plasmas if they penetrate the hot core plasma region. High $\mathrm{Z}$ materials like tungsten $(Z=74)$ on the other hand withstand very well the high heat loads in a fusion experiment and furthermore do not bind chemically the radioactive tritium used in a future fusion reactor. For this reason all plasma facing components in ASDEX Upgrade (AUG) are now coated with W. Furthermore, the divertor material in ITER will also be W. The amount of W reaching the core region does not only depend on the amount of $\mathrm{W}$ released from the wall, but also on the 'divertor retention' $R=S_{d i v} / S_{m c}$, i.e. the capability of the divertor to screen the core from impurities $S_{d i v}=\Phi^{\text {core }} / \Phi^{d i v}$ normalized to the screening factor $S_{m c}=\Phi^{\text {core }} / \Phi^{m c}$ in the main chamber. $\Phi^{\text {core }}$, $\Phi^{d i v}$ and $\Phi^{m c}$ are the impurity fluxes into the core, divertor and main chamber respectively. Spectroscopic measurements reveal that the $\mathrm{W}$ sputtering flux from divertor, although by far the largest source [1], has a relatively small impact on the central W concentration compared to that of main chamber sources indicating that $\mathrm{R}$ is high for $\mathrm{W}$ in $\mathrm{AUG}$.

In addition to simulating this effect (cf. the following two sections) in Sec. 4 we will compare our results to experiments with a massive introduction of $\mathrm{W}$ originating from a melting $\mathrm{W}$ pin in the divertor on one hand and a moderate injection of $\mathrm{W}$ in the main chamber by laser ablation on the other.

\section{Simulation of an AUG divertor discharge}

Although a tokamak is toroidally symmetric to a high degree, certain phenomena break this symmetry and require a fully $3 \mathrm{D}$ treatment of the problem. The localized injection of impurities is such a phenomenon. For this reason all the simulations presented in the following are carried out by means of the Edge Monte Carlo 3D (EMC3) - Eirene code package. Its working principle, as well as the details of the code are described in [2]. EMC3 solves Braginskii's equations in 3D and is self consistently coupled to Eirene, which solves the kinetic equation for neutral particles. Drifts are not included in the code so far. Although EMC3 also applies Monte Carlo techniques, it has to be pointed out that the plasma is described by a fluid approach. Before we address the transport of impurities in the plasma in the next section, we describe the simulation of the deuterium plasma background in this section.

For the simulation of an AUG divertor discharge EMC3-Eirene uses a grid consisting of 3 zones, core, SOL and private flux region (PFR), as shown in Fig. 1. Apart from the outermost parts of the grid that are defined for the neutrals only, the grid is aligned to the magnetic surfaces. In order to keep the computational effort small, the toroidal extension of the computational domain has so far been limited to only one sector, i.e. $2 \pi / 16$. From the three configurations we describe in the following the simulation of the non-detachment L-mode AUG plasma pulse \# 25460 at $1.7 \mathrm{~s}$ agrees best with the experiment. This discharge, which we will also refer to as configuration I, was heated by $P_{E C R H}=0.75 \mathrm{MW}$ of ECRH and $P_{O H}=0.7 \mathrm{MW}$ of ohmic power yielding in equilibrium (the stored energy in the core plasma is observed to be constant) a power flux into the edge plasma of $P_{\text {in }}=P_{E C R H}+P_{O H}=1.45 \mathrm{MW}$. The plasma current is $I_{p}=1 \mathrm{MA}$, the toroidal magnetic field at the magnetic axis $B_{t}=-2.5 \mathrm{~T}$ and $q_{95}=-4.1$. In order to match the density and temperature profiles measured by the Lithium beam and the electron cyclotron emission (ECE) diagnostics in the main chamber (cf. Fig. 2) we assumed (anomalous) parti- 


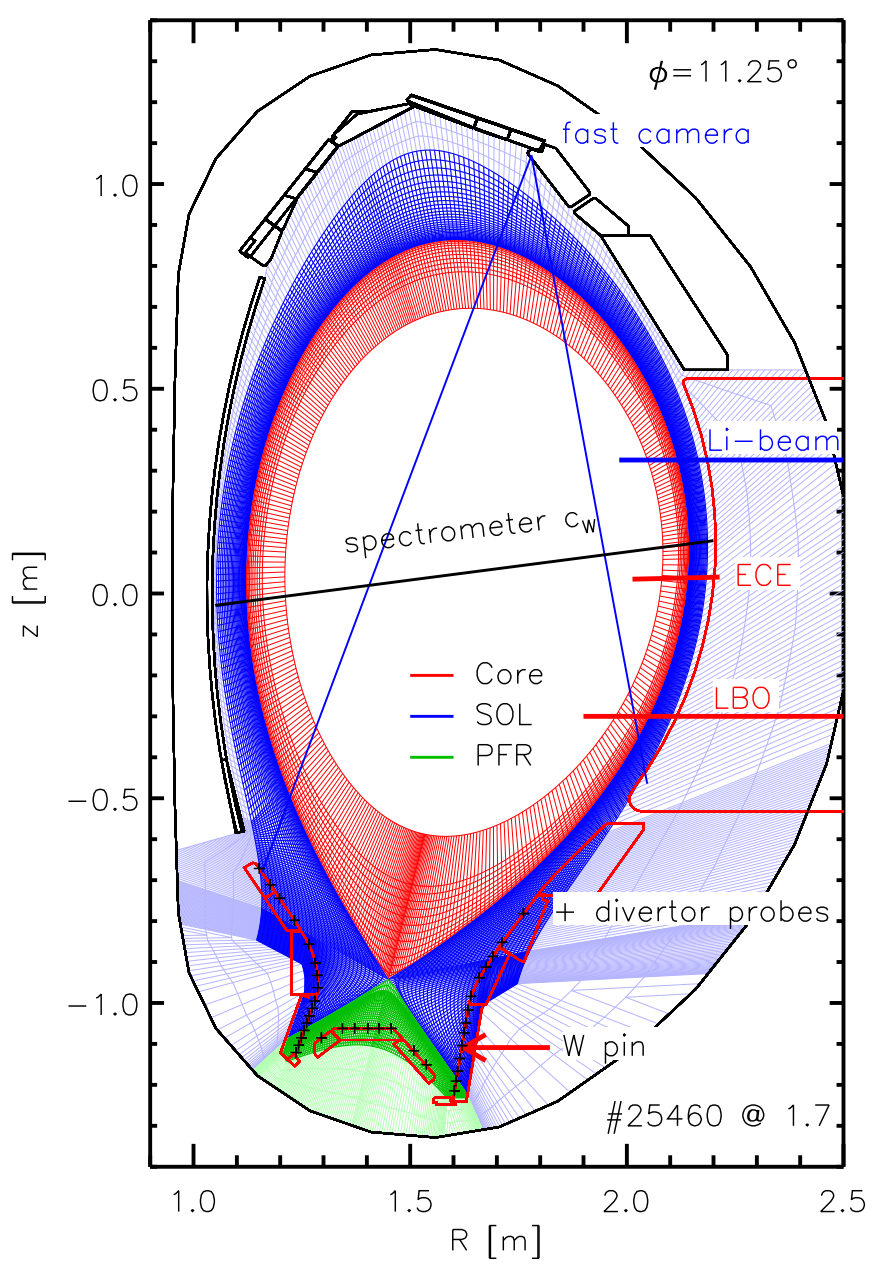

Figure 1: Cross section of the computational grid at $\phi=11.25^{\circ}$ used by EMC3Eirene to simulate the AUG divertor discharge 25460 at $1.7 \mathrm{~s}$. The grid is divided into 3 zones, 'core', 'scape-off layer' (SOL) and 'private flux region' (PFR). Tungsten impurities can artificially be introduced into the plasma by means of a melting W pin and by means of the laser blow-off (LBO). Their concentration in the core is measured spectroscopically.

cle and heat diffusion coefficients of $D_{\perp, e}=D_{\perp, i}=0.25 \mathrm{~m}^{2} / \mathrm{s}$ and $\chi_{\perp, e}=\chi_{\perp, i}=2.5 \mathrm{~m}^{2} / \mathrm{s}$, respectively, a separatrix density of $n_{\text {sep }}=1.2 \cdot 10^{19} \mathrm{~m}^{-3}$ and that the electrons receive $70 \%$ of the heating power $\left(P_{e}=0.7 P_{i n}, P_{i}=0.3 P_{i n}\right)$. The major part of this power is finally deposited on the divertor target plates (where heat sheath transmission coefficients $\gamma_{i}=2.5$ and $\gamma_{e}=4.5$ are assumed) while ionization processes of the neutrals in the SOL consume another fraction of the input energy. Since we neglect the radiative losses (measured to be $P_{\text {rad }}=0.5 \mathrm{MW}$ ) so far we somehow overestimate the power going onto the divertor tiles. Nevertheless, the measured fluxes agree relatively well with the simulated ones. Fig. 3 shows ion saturation currents measured by the divertor Langmuir probes together with the prediction of EMC3-Eirene. The density shows a similar agreement although the asymmetry between the high field side (HFS) and the low field side (LFS) is less pronounced in the simulation. At a later phase $(3.3 \mathrm{~s})$ of the discharge (configuration II) the separatrix density has increased to $n_{e}^{\text {sep }}=3.19 \cdot 10^{19} \mathrm{~m}^{-3}$ (while the other parameters remain essentially the same) and, as often

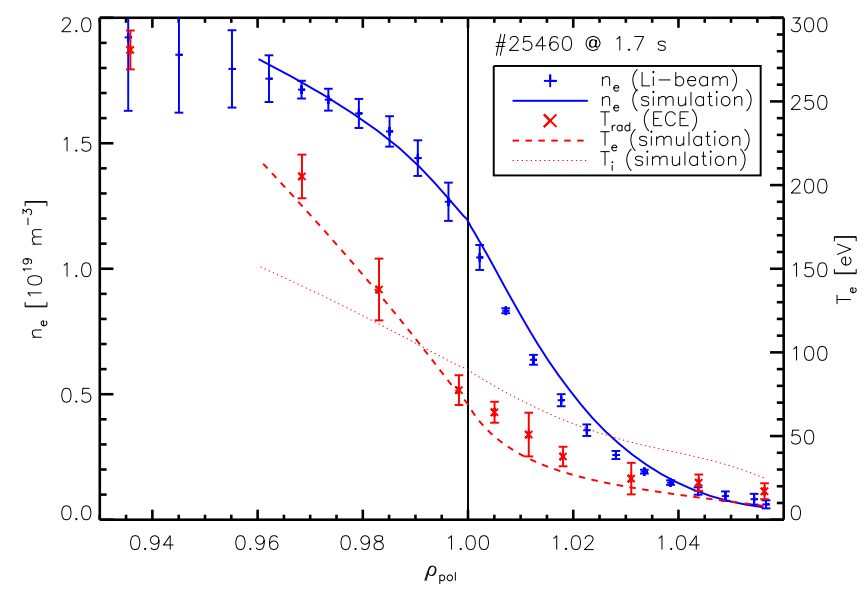

Figure 2: Density (blue) and temperature (red) profiles in the main chamber measured by the Li-beam and by the electron cyclotron emission (ECE) diagnostics (data points) and simulated by EMC3-Eirene (lines).

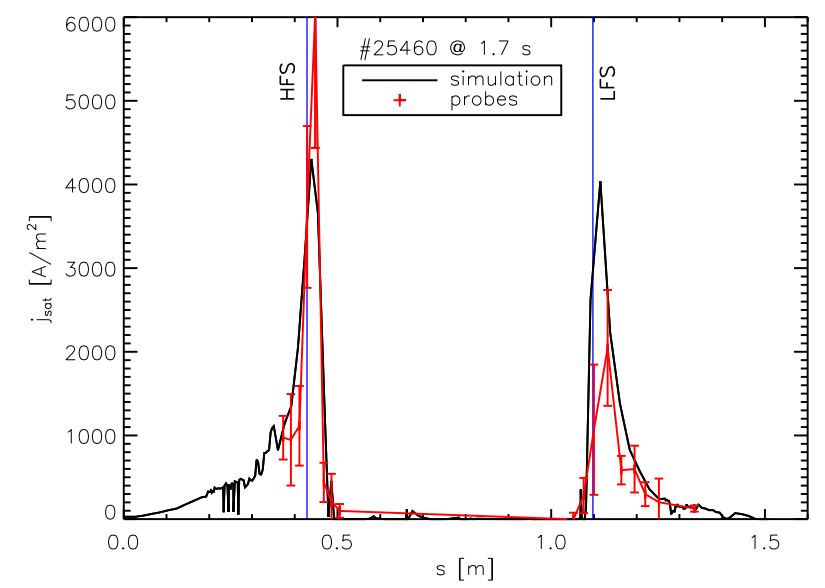

Figure 3: Current density onto the divertor target plates measured by Langmuir probes (red data points) compared to the simulation of EMC3-Eirene (black curve). $s$ defines the position along a curved path following the divertor contour, the blue lines indicate the nominal strike point positions.

observed for these conditions, detachment occurs in the divertor. Detachment is seen clearly in the current density measured by the Langmuir probes which reduces to almost zero at the HFS while its peak value shifts outward on the LFS. Although EMC3-Eirene is able to simulate the profiles in the main chamber reasonably well in this later phase, it cannot explain this behavior in the divertor so far. This problem was studied in detail by the $2 \mathrm{D}$ code package SOLPS but remains unresolved so far [3].

Besides these two configurations a third (III) was simulated. Configuration III is the AUG H-mode discharge \# 25513 at 2.2 $\mathrm{s}$ heated by significantly more power than I and II $\left(P_{N B I}=5\right.$ $\mathrm{MW}, P_{E C R H}=0.7 \mathrm{MW}, P_{O H}=0.2 \mathrm{MW}, P_{\text {rad }}=-2.2 \mathrm{MW}$, $I_{P}=0.8 \mathrm{MA}, B_{t}=-2.5 \mathrm{~T}$ and $q_{95}=-5$ ) also with clearly pronounced detachment. This discharge is a reference for \# 25514, which was dedicated to the $\mathrm{W}$ impurity transport experiments described in Sec. 4. With radially constant transport coefficients and the physics currently included in the code it is not surprising 
that the disagreement between simulation and experiment unfortunately is largest in the case of configuration III. Nevertheless, a 3D plot of the ion current density $j_{i}=e n_{i} M c_{s}$ is shown in Fig. 4 (a). Due to the Bohm condition, strong flows onto the target plates are found in the divertor, which are responsible for strong friction forces acting on impurities released from the divertor and therefore the strong retention effect addressed in the following section.

\section{Simulation of a localized $W$ impurity injection}

For the impurity transport simulation EMC3-Eirene uses a similar, but simplified set of equations as in the case of the deuterium plasma background. Instead of solving the energy equation the impurity ions are assumed to be in thermal equilibrium $T_{Z}=T_{i}$ with the deuterium ions. The continuity and momentum equations for the 75 charge states $z$ are given by Eqn. 7 and 8 in [2]. We assume $D_{\perp, Z}=D_{\perp, i}$ and determine the source terms for $\mathrm{W}$ due to ionization and recombination $S_{Z \rightarrow Z^{\prime}}\left(n_{e}, T_{e}\right)$ and $R_{Z \rightarrow Z^{\prime}}\left(n_{e}, T_{e}\right)$ from the data given in [4] modified by the empirical correction factors of [5]. Since the amount of impurities and therefore their radiated power is small, their action on the plasma background can be neglected. This 'trace limit', however, is not a principle limitation for EMC3-Eirene.

Fig. 4 (b) - (d) show the total W density $n_{W}=\sum_{i=0}^{74} n_{W^{i+}}$ distribution during an injection of $\Phi_{i n j}=6.2 \cdot 10^{18}$ neutral atoms $/ \mathrm{s}$ $(=1 \mathrm{~A} / \mathrm{e})$ for configuration III. While in (b) the injection was in the main chamber, the source was placed in the divertor (c) at the strike point $(\Delta s=0)$ on the LFS and (d) $\Delta s=6 \mathrm{~mm}$ above it. The $\mathrm{W}$ density at the separatrix was found to be $n_{W}^{\text {sep, } m c}=4.7 \cdot 10^{17} \mathrm{~m}^{-3}$ in (b), $n_{W}^{\text {sep,div }}=1.1 \cdot 10^{16} \mathrm{~m}^{-3}$ in (c) and $n_{W}^{\text {sep,div }}=1.0 \cdot 10^{17} \mathrm{~m}^{-3}$ in (d). Since in the stationary case the seperatrix $\mathrm{W}$ density, the total number of $\mathrm{W}$ atoms in the core and the $\mathrm{W}$ influx into the core are proportional to each oter $\left(n_{W}^{\text {sep }} \propto N_{W}^{c o r e} \propto \Phi_{W}^{\text {core }}\right)$, the ratio

$$
R=\frac{n_{W}^{\text {sep, } m c}}{n_{W}^{\text {sep, div }}}
$$

can be identified as the divertor retention given in the introduction. Comparing (c) and (b) we obtain a value of $R(\Delta s=0)=$ 40 at the strike point. Only $6 \mathrm{~mm}$ above it decreased almost one order of magnitute, $R(\Delta s=6 \mathrm{~mm})=5$. Due to the fact that the simulation also shows a strong dependence on $n_{e}$ and $T_{e}$ (i.e. on collisionality) and that neither detachment nor transient events like ELMs are described sufficiently well by EMC3-Eirene, the uncertainty of $R$ is very high.

\section{W transport experiments at AUG}

The capability of the divertor to shield tungsten and other impurities from the core plasma was also studied experimentally at AUG. Geier et al. [6] performed experiments with a tungsten carbonyl sublimation probe, from which they derived a moderate divertor retention of $\mathrm{R}=17$. Recently, further experiments were carried out by Krieger et al. [7], in particular with the aim to investigate the impact of a massive injection of $\mathrm{W}$ impurities due to a melting divertor tile on the stability of the discharge. For this purpose a $\mathrm{W}$ pin, with $1 \times 1 \mathrm{~mm}^{2}$ cross section and $3 \mathrm{~mm}$ long, was exposed to the plasma above the LFS strike point. Moving the strike point onto the pin for a certain time interval(2.1-2.9 s) in the discharge (\#25514) leads to a significant increase of the power influx and a melting of the pin. In the same discharge $\mathrm{W}$ impurities were also injected into the main chamber plasma by means of a 'laser blow-off' (LBO). This technique consists in ablating a thin layer $(0.5 \mu \mathrm{m})$ of $\mathrm{W}$ from a glass plate by means of a high power ( $0.6 \mathrm{~J}$ in $10 \mathrm{~ns})$ laser (diameter $4 \mathrm{~mm}$ ). Both events were recorded in the visible spectral range by a fast camera with a vertical view (cf. Fig. 1) into the vacuum chamber. The $\mathrm{W}$ concentration $c_{W}$ in the core is measured spectroscopically from the quasi-continuum emission at $5 \mathrm{~nm}$ and the spectral line at $0.794 \mathrm{~nm}$ emitted from Ni-like $\mathrm{W}^{46+}$ (cf. [5]).

Assuming that none of the ablated $\mathrm{W}$ atoms is ionized and a characteristic density of $\rho_{W}=19.25 \cdot 10^{3} \mathrm{~kg} / \mathrm{m}^{3}$ an amount of $121 \mu \mathrm{g}$ or $N_{W}^{i n j, L B O}=4 \cdot 10^{17} \mathrm{~W}$ atoms is injected into the main chamber. The mass loss of the tungsten pin due to the erosion on the other hand was measured to be $20.6 \mathrm{mg}$ or $N_{W}^{E R O}=6.7 \cdot 10^{19} \mathrm{~W}$ atoms, i.e. more than two orders of magnitude more. However, not all of these $\mathrm{W}$ atoms ionize. It was observed by the fast camera that a significant fraction of the material stays in the divertor in form of liquid tungsten droplets. Details of the analysis of the data from the fast framing camera, as well as the result that between $20 \%$ and $40 \%$ of the $\mathrm{W}$, i.e. $N_{W}^{\text {inj,pin }}=1.3 \ldots 2.7 \cdot 10^{19}$ particles, get into the divertor plasma as atomic/ionic impurities are found in [7].

Fig. 5 shows $c_{W}$ in the main chamber. The red curve represents the situation with a $\mathrm{W}$ pin, while the blue curve corresponds to the reference discharge (\#25513) without a pin. Later, tungsten was injected at the mid-plane by means of the laser blow-off in both discharges. Assuming as a OD model that the total number of $\mathrm{W}$ ions in the core $N_{W}=c_{W} \bar{n}_{e} V_{\text {core }}$ is determined by the core influx $\Phi_{\text {in }}$ according to $\dot{N}_{W}=\Phi_{i n}-N_{W} / \tau_{W}$. The confinement time $\tau_{W}=60 \mathrm{~ms}$ for $\mathrm{W}$ is determined accurately from the decay time after a LBO, the number of $\mathrm{W}$ ions penetrating the core during the time interval $t_{a} \ldots t_{b}$ is given by

$$
N_{W}^{\text {core }}\left(t_{a}, t_{b}\right)=\bar{n}_{e} V_{\text {core }}\left(c_{W}\left(t_{b}\right)-c_{W}\left(t_{a}\right)+\frac{1}{\tau_{W}} \int_{t_{a}}^{t_{b}} c_{W}(t) d t\right)
$$

The number of $\mathrm{W}$ atoms originating from the pin that penetrate the core are then given by $N_{W}^{\text {core } \text { pin }}=N_{W}^{\text {core }}-N_{W, \text { ref }}^{\text {core }}=7.9 \cdot 10^{16}$, where $t_{a}=2.07 \mathrm{~s}$ and $t_{b}=2.64 \mathrm{~s}$. Those originating from the LBOs are on the other hand $N_{W}^{\text {core } L B O}=3.7 \cdot 10^{16}$, i.e. about a factor of 2 less. This enables us to determine the screening factors

$$
S_{d i v}=\frac{N_{W}^{i n j, p i n}}{N_{W}^{c o r e, p i n}}=260 \text { and } S_{m c}=\frac{N_{W}^{i n j, L B O}}{N_{W}^{c o r e, L B O}}=11
$$

for the divertor and the main chamber, respectively. From the ratio of the two we obtain the experimental divertor retention

$$
R_{\text {exp }}=\frac{S_{d i v}}{S_{m c}}=25
$$



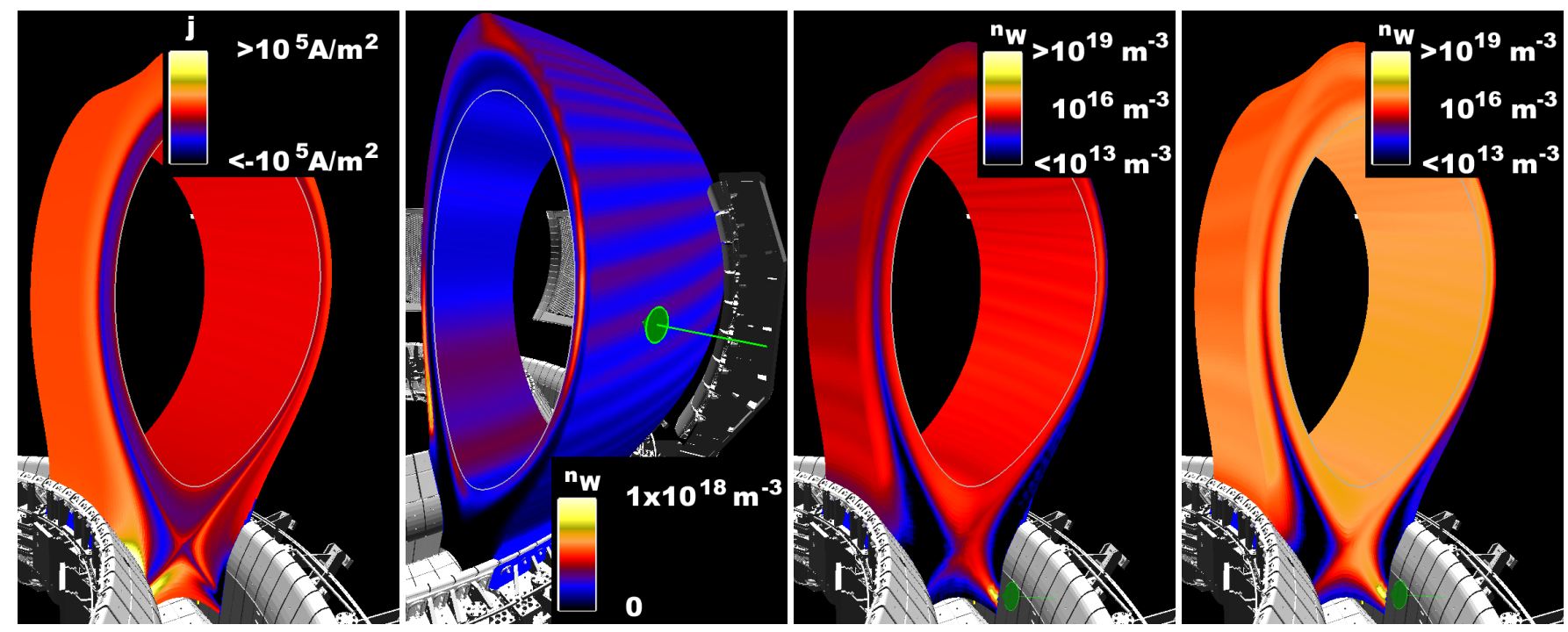

Figure 4: (a) flux density in the partially detached AUG H-mode discharge \# 25513 at 2.2 s dedicated to W transport experiments. (b) W distribution (sum over all ionization states) in the same plasma, with a localized tungsten source $\left(\Phi_{i n j}=6.2 \cdot 10^{18}\right.$ atoms/s) in the main chamber. (c) W distribution with the same amount of $\mathrm{W}$ atoms injected in the same discharge but at the strike point. (d) same as (c) but with the source shifted $\Delta s=6 \mathrm{~mm}$ upward. The injection position is indicated by a green arrow in all cases (b)-(d). Note that in (c) and (d) a logarithmic scale was used.

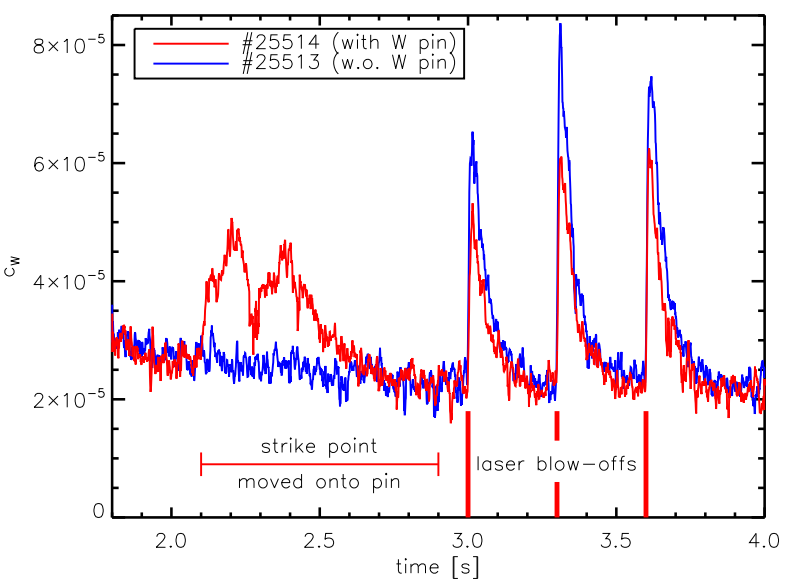

Figure 5: Spectroscopical measurement of the $\mathrm{W}$ concentration in the AUG main chamber. At the time $2.1 \mathrm{~s}$ the strike point is moved onto a $\mathrm{W}$ pin provoking a strong local erosion of material for the next $0.5 \mathrm{~s}$. At the times $3.0 \mathrm{~s}$, $3.3 \mathrm{~s}$ and $3.6 \mathrm{~s}$ tungsten is injected into the main chamber by a high power laser ablating the material from a glass plate. The blue curve shows the same signal for a reference discharge without W pin (but with the same laser blow-offs)

\section{Conclusions and outlook}

For the first time the Edge Monte Carlo 3D (EMC3) - Eirene code package was used to simulate ASDEX Upgrade (AUG) divertor discharges. Good agreement between the simulation and the experimental data in the main chamber as well as in the divertor was found for (I) a non-detached L-mode. In the case of a detached L-mode (II) and in particular of a partially detached H-mode (III) the profiles predicted by EMC3-Eirene at the target plates differ significantly from the experimental ones. Furthermore the code was applied to study the transport of $\mathrm{W}$ impurities injected at different positions in the plasma. For configuration III with a source exactly at the strike point a divertor retention of $R=40$ was found. This value, however, has a high uncertainty since the transport depends very sensitively on the distance of the injection position to the strike point and also on density and temperature, i.e. on collsionality. The results are compared to experiments with $\mathrm{W}$ impurities originating from a melting $\mathrm{W}$ pin in the divertor on one hand and injected into the main chamber by means of a laser blow-off on the other. While the screening factor for the divertor source is $S_{d i v}=260$ it is $S_{m c}=11$ for the main chamber source. In view of the uncertainties in both the experiment and the simulation the retention $R=S_{d i v} / S_{m c}=25$ is not inconsitent with the simulation.

The next step will be to solve the mentioned problems for high density discharges. Furthermore, we will extend the computational domain toroidally to account for more of the three dimensionality of the problem and simulate the impurity transport time dependently to facilitate the comparison with the experiment. A detailed study of the relevant parameters $\left(n_{e}, D_{\perp, W}\right.$ and source position) is foreseen. On the experimental side we are planning to vary the separatrix density in order to test the predicted dependency for $\mathrm{R}$.

\section{References}

[1] R. Dux et al., J. Nucl. Mater. (2009)

[2] Y. Feng et al., Contrib. Plasma Phys. 44, No. 1-3, 57 - 69 (2004)

[3] Wischmeier et al. J. Nucl. Mater. 390-391, 250-254 (2009)

[4] S. D. Loch et al. Phys. Rev. A72 (2005) 052716

[5] T. Pütterich et al., Plasma Phys. Contr. Fusion 50 (2008) 085016

[6] Geier et al., Plasma Phys. Contr. Fusion 44 (2002) 2091-2100

[7] K. Krieger et al., submitted to J. Nucl. Mater. (2010) 